\title{
Correction to: Comprehensive analysis of adverse events associated with gastric peroral endoscopic myotomy: an international multicenter study
}

\author{
Y. Ichkhanian ${ }^{1} \cdot$ K. Vosoughi ${ }^{1}$ - M. Aghaie Meybodi ${ }^{1}$. J. Jacques ${ }^{2} \cdot$ A. Sethi $^{3}$ - A. A. Patel ${ }^{3}$ - A. A. Aadam ${ }^{4}$. J. R. Triggs ${ }^{4}$.

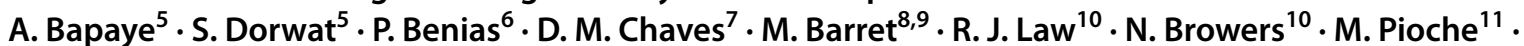

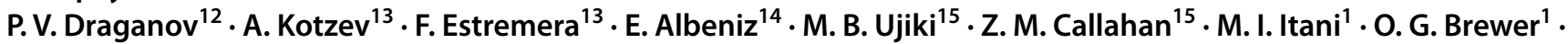 \\ M. A. Khashab ${ }^{1,16}$
}

Published online: 18 May 2020

(c) Springer Science+Business Media, LLC, part of Springer Nature 2020

\section{Correction to: Surgical Endoscopy https://doi.org/10.1007/s00464-020-07570-z}

This article was updated to correct author M.I. Itani's name in the author listing.

Publisher's Note Springer Nature remains neutral with regard to jurisdictional claims in published maps and institutional affiliations.

The original article can be found online at https://doi.org/10.1007/ s00464-020-07570-z.

\section{A. Khashab}

mkhasha1@jhmi.edu

1 Division of Gastroenterology and Hepatology, Johns Hopkins Medical Institution, Baltimore, MD, USA

2 Gastroenterology Department, Limoges University Hospital, 2 Avenue Martin Luther King, 87042 Rouen, France

3 Division of Digestive and Liver Diseases, Columbia University Medical Center, New York, NY, USA

4 Division of Gastroenterology and Hepatology, Northwestern University Feinberg School of Medicine, Chicago, IL, USA

5 Department of Digestive Diseases \& Endoscopy, Deenanath Mangeshkar Hospital and Research Center, Pune, Maharashtra, India

6 Division of Gastroenterology, Zucker School of Medicine at Hofstra/Northwell, Long Island Jewish Medical Center, Northwell Health System, New Hyde Park, NY, USA

7 Gastroenterologia, Hospital das Clinicas HCFMUSP, Faculdade de Medicina, Universidade de Sao Paulo, Sao Paulo, SP, Brazil

8 Gastroenterology Unit, Cochin University Hospital, Université Paris Descartes, Paris, France
9 Unité INSERM U1016, Université Paris Descartes, Sorbonne Paris Cité, Paris, France

10 University of Michigan Health Care System, Ann Arbor, USA

11 Service d'Hépato-gastro-entérologie, Hôpital Edouard Herriot, CHU Lyon, Lyon, France

12 Division of Gastroenterology, Hepatology and Nutrition, University of Florida, Gainesville, FL, USA

13 Clinic of Gastroenterology, University Hospital "Alexandrovska", Sofia, Bulgaria

14 Division of Gastroenterology, Complejo Hospitalario de Navarra, Pamplona, Spain

15 Section of Minimally Invasive Surgery, Department of Surgery, NorthShore University Health System, Evanston, IL, USA

16 Division of Gastroenterology and Hepatology, Johns Hopkins Hospital, Sheikh Zayed Bldg, 1800 Orleans Street, Suite 7125G, Baltimore, MD 21287, USA 